\title{
Alcune osservazioni sulle occorenze di Crux in Manilio, Seneca, Giovenale, Marziale
}

\author{
ILARIA RAMELLI *
}

\section{RIASSUNTO}

II supplizio della croce appare un tema particolarmente presente e diffuso nella letteratura latina del I secolo d.C.

Manilio, nel V libro dei suoi

Astronomica, per la prima volta attribuisce la croce ad Andromeda, operando una vistosa innovazione allinterno della tradizione mitografica. Ma anche Petronio, Lucano, Seneca, Giovenale e Marziale parlano più volte della croce. Seneca ne appare impressionato in modo speciale e Marziale cita il caso di uno schiavo crocifisso per avere incendiato Roma. Cosi Giovenale sembra fare allusione ai supplizi inflitti ai Cristiani con l'accusa di avere incendiato Roma (64 d.C.).

\section{ABSTRACT}

The torment of the cross is a theme that is particularly present in the Roman literature of the ist century A.D. Manilius for the first time ascribes the cross to Andromeda, with a remarkable innovation in the mythographic tradition. But also Petronius, Lucan, Seneca, Juvenal and Martial in several occasions mention the cross. Seneca appears particularly impressed by this torment. Martial represents a slave hanging from the cross as guilty of the fire of Rome. And so Juvenal seems to allude to the torments inflicted to the Christians as scapegoats of the fire of Rome (64 A.D.).

* Universidad católica de Milán. 
Uno studio recente ha mostrato la grande impressione che il supplizio della croce suscitò in autori del I-II secolo come Silio Italico, al punto da essere attribuita ad un eroe nazionale come Atilio Regolo, in aperto contrasto con la tradizione precedente .

Anche uno Stoico dichiarato della prima età imperiale, M. Manilio, autore degli Astronomica, in un passo celebre del $\mathrm{V}$ libro in cui è rivisitato il mito di Andromeda ${ }^{2}$, al quale è conferito un rilievo del tutto particolare ${ }^{3}$, attribuisce all'eroina, in modo completamente indipendente dalla tradizione precedente ${ }^{4}$, il supplizio della croce e manifesta sincera ammirazione per la fermezza ed il dignitoso pudore della fanciulla - la quale poi, conformemente al mito, viene infine salvata da Perseo- nell'affrontare la morte. Come è noto, infatti, Andromeda era figlia di Cefeo e di Cassiopea, che, avendo osato gareggiare in bellezza con le Nereidi, aveva irritato gli dèi marini ( «Cassiope filiae suae Andromedae formam Nereidibus anteposuit.

\footnotetext{
1 L.Cotta Ramosino, II supplizio della croce in Silio Italico (Pun. I 169-181; VI 539-544), «Aevum», 73 (1999), 93-106. Per Regolo si veda anche Flor. Epit. II 2, 25: «nec crucis supplicio deformata maiestas Reguli».

2 Per il mito si vedano K. KERÉNYl, Die Mythologie der Griechen, tr. it. Gli dèi e gli eroi della Grecia, II, Milano 19895, 61-62; P. GRIMAL, s.v. Andromeda, in Dictionnaire de la mythologie grecque et romaine, Paris $1979^{6}$, tr. it. Enciclopedia dei miti, a c. di C. CoRdí, Brescia 1987, 44-45; V. Cristobal, Perseo y Andrómeda: versiones antiguas y modernas, «Cuadernos de Filología Clásica», 23 (1989), 51-96. Per un confronto tra l'episodio ovidiano e quello maniliano: E. FLORES, Quaedam de Andromedae excursu in Manilii Astronomicon libro quinto quaestiones selectae, in Contributi di filologia maniliana, Napoli 1966, 17-34; F. DELLA CORTE, II Perseo ovidiano, in Opuscula, IV, Genova 1973, 57-63; F. PASCHOUD, Deux études sur Manilius, in RomanitasChristianitas, Berlin-New York 1982, 125-53; C. SALEMME, Introduzione agli Astronomica di Manilio, Napoli 1983 (Studi e testi dell'antichità, 15), 154-55: all'opera rinvio anche per uno studio complessivo su Manilio. Cfr. K. Wernicke, Andromeda, in PW, I, Stuttgart 1894, 2154-59; F. BolL, Sphaera, Leipzig 1903, 379, indica l'episodio di Andromeda come punto saliente dell'opera di Manilio come "Prachstück der alexandrinischen Kunst», seguito da E. NORDEN, Geschichte der römischen Literatur, Leipzig 19545, 83; cfr. W. KROLL, Studien zum Verständnis der römischen Literatur, Stuttgart 1924, 199; G.P. Goold, Perseus and Andromeda. A Myth from the Skies, "Proceedings of the African Class. Association", 2 (1959), 10-15; B.R. Voss, Die AndromedaEpisode des Manilius, «Hermes», 100 (1972), 413-34 con bibliografia; G.B.A. FletCHER, Manilius, “Durham University Journal», 34 (1973), 129-50; E. RoMANo, Andromeda: l'epillio retorico, "AAPal" 38 (1980), 213-35; K.M. Coleman, Manilius' Monster, "Hermes" 111 (1983), 226-32. Su Manilio e il problema del mito nel suo poema astronomico, oltre che al cit. SALEMME, Introduzione, 77-106 e a Voss, Die Andromeda-Episode, 421-23, rinvio a D. LIUzzı, Manilio fra poesia e scienza. Atti del Convegno, Lecce 14-16 maggio 1992, a c. di D. LIUZZ!, Lecce 1993.

3 Manilio con l'esposizione di questo mito, che è indubbiamente il più lungo excursus mitologico del suo poema, si allontana per l'unica volta dallo schema della presentazione dei 33 paranatellonta ripreso da Arato: ipotizza un influsso dell'epillio o forse dalle e̊́ $\phi \rho \alpha ́ \sigma \varepsilon t \varsigma$ delle opere didascaliche D. LiuzZI, Manilio. Astronomica, V, Galatina 1997 (Università degli Studi di Lecce. Dipartimento di Filologia Classica e Medioevale. Testi e Studi, 9), 8 n. 9; si veda anche la sintesi a 13-15

4 Cfr. per le attestazioni letterarie del mito KERÉNYI, Gli dèi e gli eroi..., 134; SALEMME, Introduzione..., 95 n. 33; DELLA CORTE, /I Perseo..., 57-63; LIUzZI, Astronomica..., V, 144.
} 
Ob id Neptunus expostulavit, ut Andromeda Cephei filia ceto obiceretur", Hyg. Fab. 64): Posidone aveva per questo inviato contro il paese di Cassiopea un'inondazione e poi un mostro marino, al quale l'oracolo comandò che venisse esposta Andromeda. Ritengo opportuno presentare il passo saliente dell'episodio di Andromeda, dove pongo in evidenza le espressioni a mio avviso più significative ed innovative rispetto alla tradizione precedente (V 538-619):

Per duras panduntur bracchia cautes;

astrinxere pedes scopulis, iniectaque vincla, et cruce virginea moritura puella pependit.

Servatur tamen in poena vultusque pudorque;

supplicia ipsa decent; nivea cervice reclinis molliter ipsa suae custos est visa figurae. (Astron. V 550-55) ${ }^{5}$

In questo passo colpisce appunto l'attribuzione all'eroina della croce, dalla quale la fanciulla pende: né nelle versioni letterarie né nell'iconografia classica pagana Andromeda è mai raffigurata appesa in croce e giustamente a mio parere il Landolfi ha notato la «novità inusitata» di questa virginea $\mathrm{crux}^{6}$. Le attestazioni letterarie precedenti a Manilio sono in effetti numerose: Apollod. mythogr. II 4, 3 presenta la scena della fanciulla avvinta agli scogli e la situa in Etiopia, paese ove regnava Cefeo padre dell'eroina; secondo altri, Andromeda si trovava invece sulla riva del mare presso l'odierna Giaffa (Pausan. IV 35, 9; Strab. XVI 2, 28; los. Bell. lud. III 9, 3). La tradizione letteraria è rappresentata anche da Eratosth. Catast.

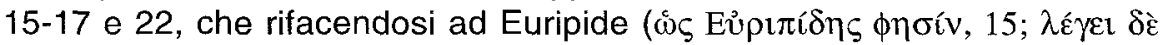

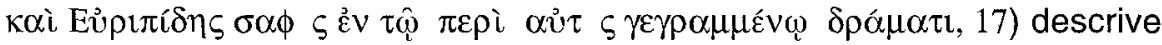
precisamente la posizione mantenuta in cielo dalla fanciulla, che è la me-

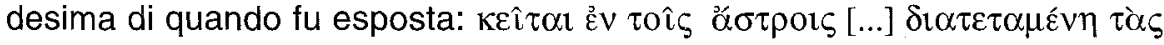

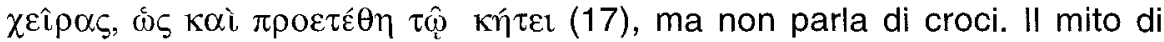
Andromeda è altresi attestato da Pherec. FGrH 3 F 12; Arat. 196; Anthol.

5 Mi baso sull'ed. Manilius, Astronomica, with an English Translation by G.P. GoOLD, Cambridge-London 1977, 344-46. Cfr. anche i vv. I 355-60; 433-37 sul medesimo episodio di Andromeda, ma qui Manilio non parla ancora di crux. Rinvio al commento di D. LiUzZI, MANILIO. Astronomica, !, Lecce 1990, Galatina 1995 (Università degli Studi di Lecce. Dipartimento di Filologia Classica e Medioevale. Testi e studi, 8), 148 ad Manil. I 354-60.

6 L. LANDOLFI, Andromeda: intreccio di modelli e di punti di vista in Manilio, "Giornale Italiano di Filologia», 45 (1993), 171-94, part. 175, seguito da LJuzzI, Astronomica..., V, 146; «si tratta di un genere di croce insolita [sic], su cui fino ad allora nessuno era stato affisso": oltre a queste brevi constatazioni, nessuno però si interroga sulle ragioni che hanno indotto Manilio ad innovare così vistosamente il mito. Anche Voss, Die Andromeda-Episode..., 414 espone: «und kettete sie in Kreuzform an», e a 416 e 419 cita ripetutamente Manil. V 552 (et cruce virginea moritura puella pependit), senza però soffermarsi oltre sul dettaglio. 
Pal. V 132; Anth. Plan. 146; Hygin. Astron. II 10; Fab. 64; Cic. Arat. 442 sgg.; Prop. II 28, 21-22; Ov. Ars I 53; II 643-44; Ep. 17, 150-52; Met. IV 663-739; IX 212, 781, che pure non fanno alcuna menzione di una qualche croce. Cratino riprese il mito di Andromeda nei $\Sigma \varepsilon p$ ípıo (Comm. Att. Fr. 175 sgg. Kock); Frinico secondo la Suda (s.v. $\Phi \rho v v i \chi o ́ s)$ aveva scritto una tragedia su Andromeda, come Sofocle, ed anche Licofrone, ma sono perdute; nell'Andromeda di Euripide, che abbiamo già incontrato citata in Eratostene e che fu molto apprezzata (schol. Aristoph. Ranae, 53; Athen. XII 537 D; Lucian. Hist. Conscr. 1; Eunap. FHG IV 38), si presentava la liberazione dell'eroina da parte di Perseo (frr. 114-56 Nauck) ${ }^{7}$ : l'immagine

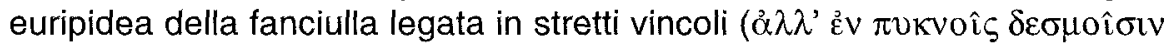

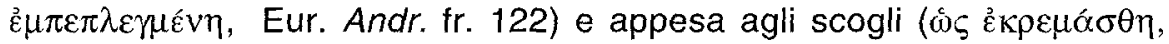
ibid.) si avvicina molto alla scena maniliana, ma in Euripide manca ogni riferimento ad una eventuale croce. Soprattutto, il modello immediato di Manilio risulta Ov. Met. IV 670-764. Nemmeno qui si trovano cenni alla croce; ci si limita a parlare di catene che stringono le braccia della fanciuIla alle dure rocce (è evidente la consonanza metrica e lessicale di Manil. V 550 con Ov. Met. IV 672, dal quale Manilio riprende pianamente la formula bracchia cautes in chiusura di verso):

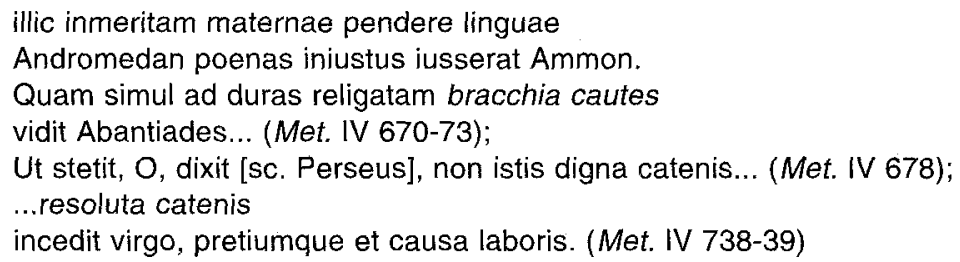

In una tanto stretta dipendenza - finanche verbale- di Manilio da Ovidio, colpisce tanto più l'innovazione del poeta astronomico rispetto ad Ovidio e, con lui, a tutta la precedente tradizione, sia letteraria, come abbiamo visto, sia iconografica, come ora vedremo.

Nelle attestazioni iconografiche, Andromeda appare sì legata per le braccia e per i piedi, e le braccia sono sovente anche divaricate, secondo

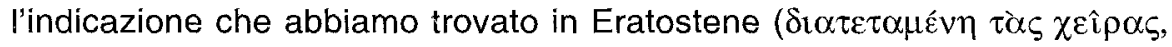
Eratosth. Cat. 17) ma in nessun caso ella pende, né tanto meno da una

\footnotetext{
7 La tragedia si apriva con un monologo di Perseo, che credeva di aver visto da lontano una figura dipinta e non una fanciulla viva (Eur. fr. 125); in un successivo dialogo tra i due Andromeda supplicava lo straniero di liberarla (Eur. fr. 132), il che avveniva, e alla fine della tragedia Atena prometteva l'assunzione fra le costellazioni ad Andromeda, Cassiopea, Cefeo e Perseo. Si veda anche T.B.L. WEBSTER, The Tragedies of Euripides, London 1967, 192-99.
} 
croce: insomma, non è mai sospesa in croce ${ }^{8}$. Manilio invece si sofferma precisamente sulla descrizione della «messa in croce», chiarissima nell'apertura delle braccia (panduntur bracchia), accanto alla quale è ricordato il fissaggio dei piedi e quindi, soprattutto, l'atto del «pendere dalla croce per morirvi sopra" (cruce moritura pependit) in cui è ritratta Andromeda, che non trova alcun riscontro nella tradizione letteraria ed iconografica precedente. Questa insistenza sul "pendere» dalla croce ritorna anche più avanti nell'episodio, sia al v. 565 (pendentia membra), sia al v. 607 (pendet). Un'altra innovazione rispetto alla tradizione letteraria del mito di Andromeda è l'ammirazione di Manilio di fronte alla dignità, al composto coraggio della giovinetta, che sa mantenere il proprio vultus ed il proprio pudor e per la quale anche quell'orribile supplizio non è vergognoso, ma decet $^{9}$. E noto infatti che la croce era sentita, oltre che come tortura atroce, anche come una orrenda infamia, un dedecus appunto ${ }^{10}$. Ma Andromeda sa mantenere il suo decus anche in croce ed è ritratta da Manilio appesa in croce con il capo reclinato (cervice reclinis). Le fonti di Manilio invece non si soffermano sul decus della fanciulla ${ }^{11}$, qui dunque tanto più significativo e paradossale in quanto Andromeda in Manilio muore sull'infamante croce; questo sembra qualcosa di più della mera esaltazione della pudicizia della fanciulla: è la paradossale trasformazione del mas-

8 Sull'iconografia pagana cfr. K. Schauendurg, Perseus in der Kunst des Altertums, "Antiquitas", Reihe 3, 1 (1960), 55-76; ID., Andromeda, in Lexicon Iconographicum Mythologiae Classicae, I, 1, Zürich-München 1981, 774-90; I, 2, ibid. 1981, 622-42, part. figg. I, 2-I, 64. Soltanto nella fig. 152 la persona di Andromeda dà l'impressione di pendere dall'alto, avendo le braccia aperte e sollevate al di sopra del capo: ma questo non indebolisce la nostra ipotesi, poiché si tratta di un rilievo sepolcrale ora al Museo Archeologico di Gap databile verso la fine del I secolo d.C., successivo quindi a Manilio. I supporti ai quali Andromeda è legata nelle pitture e nelle raffigurazioni plastiche sono quanto mai varî: si va da pali stilizzati (ad es. figg. 5-7) a colonne (figg. 9, 11, dove la donna è inscritta in un tempietto), ad una coppia di fusti arborei piantati l'uno accanto all'altro (figg.13, 17), ad un trono su cui la fanciulla è seduta (figg. 16), alla nuda roccia (figg. 19, 32).

9 Allo stesso modo per Regolo nel già citato Flor. Epit. II 2, 25: «nec crucis supplicio deformata maiestas Reguli»».

10 Un contemporaneo di Manilio, s. Paolo, designa notoriamente la croce come $\varepsilon^{\prime} \theta v \varepsilon \sigma l \mu \omega \rho i \alpha$

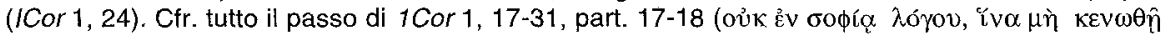

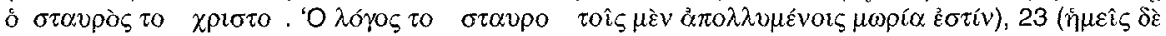

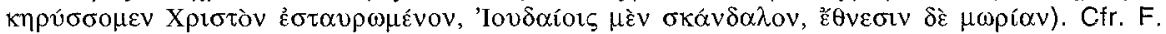
RUGgiero, La follia dei Cristiani. Su un aspetto della "reazione pagana" tra l e V secolo, pref. di M. SIMONETTI, Milano 1992, 21-52.

11 Esso se mai può richiamare it celebre episodio lucreziano del sacrificio di Ifigenia (Lucr. I 80-101: cfr. E.E. SIKES, Roman Poetry, London 1923, 179-82), però Ifigenia come è noto non muore in croce. SALEMME, Introduzione..., 113, legge alla base dell'intero episodio un linguaggio di elegia erotica (dove però non è mai questione di supplizî in croce); Lıuzzı, Astronomica..., V, 145. PASCHOUd, Deux études..., 148-53 riguardo alle fonti di Manilio sostiene che siano, oltre ad Ovidio, anche Euripide e forse l'epillio del papiro di Chicago: in questi testi non vi è cenno né alla croce né alla fermezza e al decoro della fanciulla né alla miseratio degli astanti, i tre elementi innovativi di Manilio che cerco di porre in luce nel testo. 
simo dedecus in decus. Si osservi la ripetuta designazione della morte in croce come poena (vv. 553, 574) e supplicium (v. 554) per Andromeda; ed in effetti la croce è sovente detta esplicitamente supplicium o punizione - sentita come terribile ed ignominiosa ed inflitta ai criminali per un delitto- dagli autori latini del I-II secolo, ma anche precedenti e successivi ${ }^{12}$. Eppure Andromeda non era una criminale punita per i suoi delitti; il suo era un sacrificio espiatorio e non una punizione per una colpa da lei commessa. Tenuto conto di questo, appare ancor più problematica l'attribuzione della croce alla fanciulla - come supplizio che tuttavia a lei decet- da parte di Manilio. Vi è un terzo aspetto, oltre all'introduzione della crux e, per contrasto, del decus della fanciulla, sotto il quale Manilio innova rispetto alla tradizione letteraria: la miseratio mostrata dagli astanti di fronte alla fanciulla crocifissa ${ }^{13}$ : «alcyones [...] fleveruntque tuos miserando carmine casus" (Astr. V 559-60); "Nereis [...] casus miserata tuos, roravit et undas» (ibid. 563-64). Tengo a ribadire che tutti gli aspetti del passo su Andromeda messi in luce finora appaiono deliberate innovazioni di Manilio, per le quali il poeta volutamente si discosta dalla precedente tradizione; rispetto ad Ovidio in particolare è stato notato come Manilio abbia scelto di concentrare appunto l'attenzione più sulla fanciulla e sul suo supplizio che sull'eroe ${ }^{14}$.

Gli Astronomica, come è noto, sono di difficile datazione e, se abbiamo almeno un preciso terminus post quem nel $9 \mathrm{~d}$.C., non siamo in grado di stabilire fino a quando Manilio prolungò la stesura del poema, se in età tiberiana o anche oltre ${ }^{15}$, e non siamo nemmeno sicuri che i cinque libri ri-

12 Ad es. Sen. Vit. beat. 19, 3: «cum refigere se crucibus conentur, [...] ad supplicium tamen acti stipitibus singulis pendent»; ep. 101, 12: "acutam sessuro crucem [...] differat [...] supplicii finem"; iuv. VI 219-20: “pone crucem servo'. Meruit quo crimine servus / supplicium?"; XII 104105: «multi/ committunt eadem diverso crimina fato;/ ille crucem sceleris pretium tulit, hic diadema»; Mart. Spect. 7, 7; "pendens in cruce [...] supplicium dignum tulit [...] poena fuit...»; Phaedr. III 5, 10: "poenas persolvit cruce»; Suet. Galb. 9: "cruce adfecit [...] poenam levaturus"; ancora in Flor. Epit. II 2, 25: "crucis supplicio» e Paul. Sent. V 17, 2: «summa supplicia sunt crux crematio decollatio".

13 Totalmente assente anche dal passo ovidiano delle Metamorfosi più volte citato, questa miseratio appare di tono elegiaco e comunque è un'innovazione rispetto ai modelli: LiuzzI, Astronomica..., V, 147.

14 SALEMmE, Introduzione, 154-55 e bibliografia; Paschoud, Deux études..., 125-53.

15 Sulla discussa cronologia maniliana H. WEMPE, Die literarische Beziehungen und das chronologische Verhältnis zwischen Germanicus und Manilius, «Rheinisches Museum», 84 (1935), 89-96; M. PAuER, Zur Frage der Datierung des astrologischen Lehrgedichts des Manilius, München 1951; E. Flores, Augusto nella visione astrologica di Manilio ed il problema della cronologia degli Astronomicon libri, “Annali della Facoltà di Lettere dell'Univ. di Napoli», 9 (1960-61), 5-66; E. GEBHARDT, Zur Datierungstrage des Manilius, "Rheinisches Museum», 104 (1961), 278-86; G.P.Goold, A Greek Professorial Circle at Rome, "Transactions and Proceedings of the American Phil. Association», 91 (1961), 168-92; G.B.A. FLETCHER, Manilius, "Durham University Journal», 65 
mastici costituiscano la totalità del poema ${ }^{16}$. Non è dunque possibile datare con precisione il passo su Andromeda del $\mathrm{V}$ ed ultimo libro, se non da Tiberio in avanti. Ma possiamo dire con sicurezza che l'attenzione posta al supplizio della croce da Manilio trova, nella letteratura e nella storia, ampî riscontri contemporanei e di poco successivi che possono contribuire ad illuminare la decisione del nostro poeta di introdurre nell'antico mito un supplizio che all'epoca sua era divenuto al centro dell'attenzione.

La morte in croce è ampiamente presente nella letteratura latina del I secolo e degli inizî del II, in primo luogo nell'ambito del romanzo come in Petronio, nel celebre episodio dei crocifissi della novella della Matrona di Efeso, dove peraltro si è potuta vedere una possibile parodia della morte in croce e della resurrezione di Gesù Cristo; anzi, questo tema della crocifissione diviene addirittura topico nel romanzo greco a partire da un contemporaneo di Petronio, Caritone di Afrodisia, ed è presente anche nell'altro romanziere latino, Apuleio ${ }^{17}$. Ma in questa sede vorrei occuparmi degli autori latini del I sec. ex. - II in ${ }^{18}$, nei quali il sup-

(1973), 129-50; Goold, Introduction a Manilius, Astronomica, xi-xiv; L. CICU, La data dei Phaenomena di Germanico, "Maia», n.s., 31 (1979), 139-44; D. Liuzzı, Manilio, Astronomica, I. I: destinatario ed epoca di composizione, «Miscell. Class. Mediev., Quaderni della Facoltà di Magistero dell'Univ. di Lecce", 2 (1979), 127-39; SALEMME, Introduzione, 68-72; 150-51.

${ }_{16}$ Gerberto (Ep. 8 Havet) attesta otto libri per Manilio: questa notizia è accolta da $P$. THIELSCHER, Ist M. Manilii Astronomicon libri V richtig?, «Hermes», 84 (1956), 353-72. Secondo D.B. GAIN, Gerbert and Manilius, «Latomus», 29 (1970), 128-32, i tre libri perduti sarebbero stati precisamente tra il IV ed il V. Cfr. O. REEH, Interpretationen zu den Astronomica des Manilius mit besonderer Berücksichtigung der philosophischen Partien, Marburg 1973, 154; S. CostanzA, Ci fu un sesto libro degli Astronomica di Manilio?, in Filologia e forme letterarie. Studi offerti a $F$. della Corte, III, Urbino 1987, 223-63. Depongono inoltre a favore di una lunga gestazione e di prolungati tempi di stesura dell'opera l'augurio iniziale del poeta di vivere fino alla vecchiaia (benché si tratti di un tema in parte topico) e la profonda evoluzione riscontrata dai critici nel corso del poema maniliano: Manil. | 114-117: «Faveat magno Fortuna labori, / annosa et molli contingat vita senecta, / ut possim rerum tantas emergere moles / magnaque cum parvis simili percurrere cura». Per l'evoluzione stilistica SALEMME, Introduzione, 129 e passim.

17 Anche altrove in Petronio ricorre la menzione della croce: Petr. 58, 2: "crucis offla, corvorum cibaria"; 111: "latrones iussit crucibus affigi»; 137: "si magistratus hoc scierint, ibis in crucem». Sulle crocifissioni e le possibili allusioni al Cristianesimo nella novella della Matrona di Efeso cfr. il mio Petronio e i Cristiani: allusioni al vangelo di Marco nel Satyricon?, "Aevum", 70 (1996), 75-79, accettato da C.P. THIEDE, Ein Fisch für den römischen Kaiser, München 1998, 98-123 e da G.G. Gamba, Petronio Arbitro e i Cristiani. Ipotesi per una lettura contestuale del Satyricon, Roma 1998 (Biblioteca di Scienze religiose, 141), 349-62, su cui cfr. però la mia recensione ("Aevum», 73, 1999, 207-10); per Caritone THIEDE, Ein Fisch, 128-35. Apul. Met. VI 31: i briganti vogliono uccidere la fanciulla scappata loro a dorso d'asino: «variae fuere sententiae, ut [...] tertius patibulo suffigi iuberet», ma uno consiglia "nec feras nec cruces nec ignes nec tormenta", bensi un supplizio ancor più crudele che poi viene descritto minutamente.

${ }_{18}$ Per Sil. I 181 cfr. Cotta Ramosino (cit. n. 1); Plin. Nat. Hist. XIV 12 riporta un aneddoto sull'altezza straordinaria degli olmi di Ariccia dai quali pendevano viti: «ferunt legatus regis Pyrrhi Cineam facete lusisse in austeriorem gustum vini, merito matrem eius pendere in tam altam crucem"; in XXXVI 107 la croce è usata da Tarquinio Prisco (però post mortem, il caso è diverso) 
plizio della croce si presenta con sorprendente insistenza e i quali si mostrano talora, come nel caso di Seneca, di Marziale o di Giovenale, come vedremo, particolarmente impressionati dalla facilità e dalla atrocità della condanna ${ }^{19}$.

Seneca, che prima ancora di Silio Italico attribuisce a Regolo la croce a differenza di tutti gli autori precedenti (Ep. 98, 12; Prov. 3) ${ }^{20}$, appare in effetti impressionato in modo particolare dall'apparato spettacolare degli strumenti di morte, fra cui specialmente la stessa croce, più ancora che in alcune opere precedenti ${ }^{21}$, soprattutto nelle Epistulae ad Lucilium, le quali come è noto risalgono agli anni successivi al ritiro politico (62-64 d.C.): Ep. 14, 4-6: «ingens alterius mali pompa est [...] cogita hoc loco carceres et cruces et eculeos et uncum [...] varietas magna et apparatus terribilis est [...] plus agit tortor quo plura instrumenta doloris exposuit [...] haec ut magna bella aspectu paratuque vicerunt». Straordinaria - quasi esasperata, diremmo- è l'insistenza di Seneca sul supplizio della croce, certo visto direttamente e descritto a lungo, in maniera viva ed icastica:

illud Maecenatis turpissimum votum, quo non recusat [...] novissime acutam crucem: '[vitam] mihi, vel acuta si sedeam cruce, sustine' [...] contemptissimum putarem si vivere vellet usque ad crucem: suffigas licet et acutam sessuro crucem subdas' [...] inter supplicia tabescere et perire membratim et

come deterrente dal suicidio per gli sfiniti costruttori della Cloaca Massima, che altrimenti si sarebbero dati la morte in massa: «cum id opus Tarquinius Priscus plebis manibus faceret, essetque labor incertum maior an longior, passim conscita nece Quiritibus taedium fugientibus, [...] remedium invenit [...] ut omnium ita defunctorum corpora figeret cruci spectanda civibus simul et feris volucribusque laceranda»: è dunque sottinteso che il supplizio della croce è sentito come talmente infamante da risultare peggiore della morte stessa. Le varie altre attestazioni di crux nei latini del i ex. - Il in. sono nel corso del presente lavoro e delle note (ho svolto infatti uno spoglio sistematico di tutte le attestazioni presenti sia nel ThL/ sia nel $C D$-Rom degli autori latini del Packard Humanities Institute).

${ }_{19}$ Anche Svetonio attesta l'atrocità del supplizio della croce, quando considera un atto di clemenza quello di Cesare: «piratas, a quibus captus est, cum in dicionem redegisset, quoniam suffixurum se cruci ante iuraverat, iugulari prius iussit, deinde suffigi» (Caes. 74) e un atto di sarcastica crudeltà quello di Galba: «tutorem, quod pupillum [...] veneno necasset, cruce adfecit [...] civem Romanum se testificanti, quasi solacio et honore aliquo poenam levaturus, mutari multoque praeter ceteras altiorem et dealbatam statui crucem iussit” (Galb. 9), come pure quello di Domiziano, che oltre a far uccidere Ermogene per presunte allusioni irrispettose fece crocifiggere anche i copisti: «librariis etiam, qui eam descripserant, cruci fixis» (Dom. 10).

20 Il primo passo è citato anche da CotTa Ramosino, II supplizio della croce..., 99.

21 Colpisce specialmente Sen. ad Marc. 20, 3: «video istic cruces non unius quidem generis [...] capite quidam conversos in terram suspendere [è il supplizio inflitto anche a S. Pietro], alii per obscaena stipitem egerunt, alii brachia patibulo explicuerunt [...] at video et mortem»; cfr. anche Clem. 1 23: "pessimo vero loco pietas fuit, postquam saepius culleos vidimus quam cruces", | 26: “crudelitatem privatorum quoque serviles manus sub certo crucis periculo ultae sunt». 
totiens per stilicidia emittere animam [...] adactus ad illud infelix lignum [...], cui multae moriendi causae etiam citra crucem fuerant, trahere animam tot tormenta tracturam (Ep. 101, 10-14) 22 .

Anche Marziale ${ }^{23}$ presenta un passo che appare di notevole interesse e nel quale egli sembra colpito dalla spettacolarizzazione del supplizio della croce, subito da un condannato addirittura sulla scena di un mimo, il celebre Laureolus, che rappresentava appunto la crocifissione di uno schiavo:

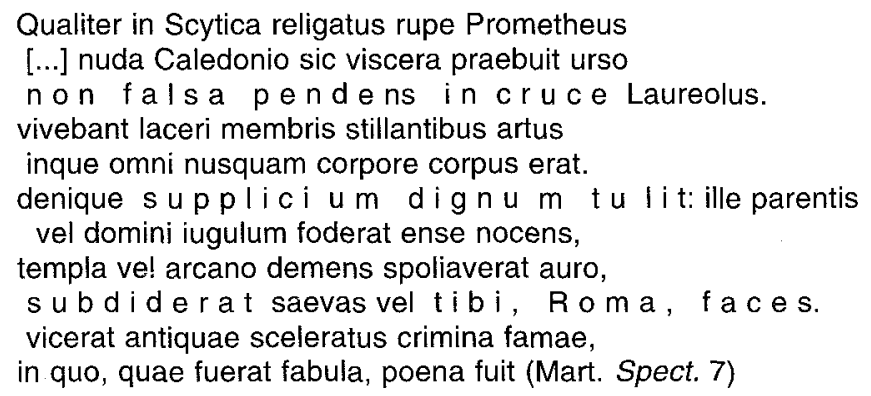

Come si nota, fra le accuse supposte dal poeta alla base della condanna a morte, accanto all'assassinio del padrone e alla spoliazione di templi, vi è quella di aver incendiato Roma («subdiderat saevas vel tibi, Roma, faces»): sappiamo da Tacito che una serie di crocifissioni dietro accusa di aver incendiato l'Urbe furono eseguite a Roma -e segnatamente contro i Cristiani, incriminati da Nerone come capri espiatorî (Tac. Ann. XV 44) ${ }^{24}$-, in occasione del celebre incendio neroniano del 64, un episodio che Marziale certamente

22 Sul costante timore della morte in Seneca e sulla sua tematizzazione: Sénèque, Entretiens. Lettres à Lucilius, a c. di P. VEYNE, Paris 1993, tr. it. P. VEYNE, Seneca, Bologna 1999 (Saggi 490), 24647 e passim. La croce è a tal punto oggetto delie meditazioni di Seneca da divenire anche fulcro di una metafora di carattere etico in Vit. beat. 19, 3 come "croce del peccato", poiché i peccati crocifiggono chi li commette: «Cum refigere se crucibus conentur [sc. sapientes], in quas unusquisque vestrum clavos suos ipse adigit, ad supplicium tamen acti stipitibus singulis pendent; hi, qui in se ipsi animum advertunt, quot cupiditatibus tot crucibus distrahuntur. At maledici et in alienam contumeliam venusti sunt. Crederem illis hoc vacare, nisi quidam ex patibulo suo spectatores conspuerent!». Una metafora affine si trova nella letteratura cristiana: se per Seneca i peccati inchiodano gli uomini alla croce, nel Nuovo Testamento Cristo ha preso su di sé i nostri peccati e li ha inchiodati nel legno della croce (lo nota F.L. DE LEÓN, De los nombres de Cristo, 7: «se escribe en otro lugar de Cristo: 'que nuestros pecados todos los subió en sí, y los enclavó en el madero'”; cfr. Rm 6,6; Gal 2,20 e soprattutto Col 2, 13-14).

23 Marziale parla dell'atroce supplizio della croce anche in X 82, 5-7: «sed si non fias quadrante beatior uno/ per gemitus nostros ingenuasque cruces,/ parce, precor».

24 Da ultimo L. Koutaïssof, Néron et le supplice des chrétiens, in Rome à l'époque néronien$n e$, in corso di pubblicazione nella serie dei Neronia, i colloqui internazionali della SIEN, ricostruisce in base a Tacito e alle disposizioni del diritto romano citate dal Digesto i supplizî dei Cristiani nel 64: l'esposizione ai cani delle vittime ricoperte di pelii ferine e la crocifissione con simultanea cremazione dei condannati impiegati come torce umane. 
conosceva e al quale potrebbe alludere in questi versi. Marziale infatti, nato nel 38-40 d.C. nella Spagna Tarragonese, nel 64 era a Roma, godeva dell'appoggio della famiglia di Seneca e frequentava i circoli dell'opposizione senatoria a Nerone: è probabile che avesse assistito alle pubbliche e spettacolari esecuzioni della multitudo ingens degli incriminati (Tac. Ann. XV 44), che sapesse dell'accusa anticristiana di incendio e che fosse colpito dalla ostentata atrocità dei supplizî. Anche se egli non dice che l'uomo realmente crocifisso nel mimo Laureolus fosse un Cristiano, è comunque assai probabile, data la precisazione dell'accusa e date le circostanze biografiche di Marziale, che egli avesse in mente proprio questo episodio storico. II cosiddetto liber de spectaculis, al quale appartiene l'epigramma che ci interessa, fu composto nell' 80 , sotto Tito, per celebrare l'inaugurazione dell'Anfiteatro Flavio e venne commissionato molto probabilmente da ambienti di corte.

Prima di proseguire nell'analisi dei testi latini del periodo sopra determinato in cui è presente il supplizio della croce, è opportuno considerare le attestazioni relative alle esecuzioni degli incriminati dell'incendio romano del 64, che notoriamente erano Cristiani. Dei supplizi spettacolari e delle crocifissioni non solo di uomini, ma anche di donne e fanciulle cristiane in quella occasione ci testimoniano Clemente Romano e Tacito. II primo nella prima lettera ai Corinzi attesta che durante la persecuzione neroniana ${ }^{25}$ «per gelosia [ $\zeta \lambda \circ \zeta$ ] donne, "giovinette e fanciulle», sotto le spoglie di Dirci e di Danaidi, furono perseguitate e soffrirono oltraggi terribili ed empî per la fede" $(6,2)^{26}$. Tacito analogamente testimonia dei supplizi spettacolari e delie crocifissioni in quel medesimo frangente (Ann. XV 44):

ergo abolendo rumori Nero subdidit reos et quaesitissimis poenis affecit quos per flagitia invisos vulgus Christianos appellabat [...] igitur primum correpti qui fatebantur, deinde indicio eorum multitudo ingens haud proinde in crimine incendii quam odio humani generis convicti sunt. Et pereuntibus addita ludibria, ut ferarum tergis contecti laniatu canum interirent, aut crucibus adfixi, aut flammandis atque, ubi defecisset dies, in usum nocturni luminis uterentur. Hortos suos ei spectaculo Nero obtulerat et circense ludicrum edebat, habitu aurigae, permixtus plebi, vel curriculo insistens. Unde quamquam adversus sontes et novissima exempla meritos miseratio oriebatur ${ }^{27}$.

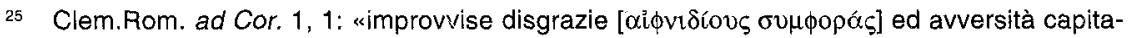
teci l'una dietro l'altra'.

${ }_{26}$ La lettura del passo è incerta: il testo di Die Apostolischen Väter, edd. F.X. FunK-K. BIHLMEYER-

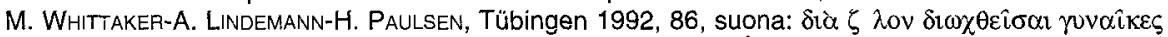

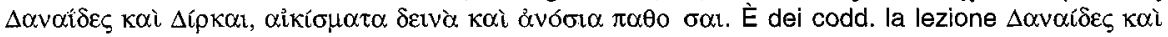

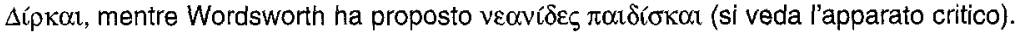

27 Su questo passo V. CAPOcCI, Per II testo di Tacito, Annales 15, 44, 4 (sulle pene inflitte ai Cristiani nel 64 d.C.), «SDHI», 28 (1962), 65-99. 
È probabile che anche diversi intellettuali di età neroniana, che allora si trovavano a Roma, siano stati impressionati dai supplizi, spesso spettacolari, degli uomini ed anche delle donne e delle fanciulle cristiane, per le quali la croce era davvero una punizione per la loro superstitio illicita, e presi dal senso di pietà (miseratio) che secondo Tacito invase gli spettatori ${ }^{28}$.

Ai medesimi episodi si riferisce forse anche, nella sua Satira I, Giovenale, che per altro in più luoghi fa menzione del supplizio della croce ${ }^{29}$. In Sat. I 155-57 invece il poeta non parla propriamente di croci, ma descrive comunque un supplizio che, anche per la precisa indicazione cronologica da lui fornita, sembra richiamare le drammatiche esecuzioni del 64. Nel contesto di un dialogo con un amico fittizio che consiglia al poeta di non scrivere nulla contro il volere dei potenti, Giovenale fa dire all'amico stesso come exemplum ammonitorio che al tempo di Tigellino si poteva finir male, come quelli che, impalati, erano usati quali torce umane nel circo: «pone Tigellinum: taeda lucebis in illa / qua stantes ardent qui fixo pectore fumant, / et latum media sulcum deducis harena" ${ }^{30}$. L'accenno ai condannati a morte bruciati come torce umane e uccisi nell'arena del circo ai tempi di Tigellino sembra rinviare precisamente ai supplizi spettacolari che furono inflitti ai presunti incendiarî durante la persecuzione neroniana nel 64, quando a fianco di Nerone era appunto Tigellino: ho posto in evidenza prima, nel passo tacitiano citato, gli espliciti accenni all'uso dei condannati come torce umane $e$ al circense ludicrum organizzato da Nerone ${ }^{31}$. È interessante allora notare qui per inciso che un supplizio altrettanto spettacolare e molto simile - fungere da torcia umana sospesi in croce- è menzionato da Lucano alla fine del suo poema, nell'incontro dei cortigiani Egiziani Achilla e Potino: "crucibus flammisque lucemus, si fuerit formonsa

28 La ricorda R. MARTIN, Le peuple de Rome au temps de Néron vu par Tacite, in Rome à l'époque néronienne. Neronia VI. Vlème colloque International de la Société Internationale des Études Néroniennes, Rome 19-23 mai 1999, ed. Y. PERRIN, in corso di pubblicazione.

29 Oltre aj già citati luv. VI 219-220 (dove è chiaramente condannata la facilità con cui i padroni potevano mandare a morte gli schiavi, senza che si rendessero conto della loro umanità: un tema sviluppato anche da Seneca); XII 104-105, si veda anche XIV 77-78: "vultur iumento et canibus crucibusque relictis ad fetus properat partemque cadaveris adfert".

30 Testo di luvenalis Saturae, edd. P. DE LABRIOLLE-F. VILLeneuve, Paris 19576. L'ultima edizione critica teubneriana (luvenalis, Saturae, ed. J. WILLIS, Stuttgardiae-Lipsiae 1997, 9) integra: «taeda lucebis in illa / qua stantes ardent qui fixo gutture fumant / <quorum informe unco trahitur post fata cadaver / et latum media sulcum deducit harena".

31 Sulla spettacolarità come cifra dell'età neroniana da ultimo L. POLVERINI, // sistema spettacolare romano nell'età di Nerone, in Rome à l'époque néronienne, cit., in corso di stampa. Un ulteriore, possibile indizio di conoscenza del Cristianesimo da parte di Giovenale sembrerebbe poi la critica (Sat. XIV 120-21) all'avido secondo cui esistono «nulla exempla beati pauperis": che secondo Giovenale esistano, al contrario, esempi di pauperes beati offre un apparente parallelo -che naturalmente resta da verificare- con la beatitudine evangelica beati pauperes. 
soror» (X 365) ${ }^{32}$. Lucano scriveva il $X$ ed ultimo libro rimastoci del suo poema verso la fine ormai della sua vita, che come è noto egli terminò nel 65 d.C. su ordine di Nerone (Tac. Ann. XV 70) durante la repressione seguita alla congiura pisoniana: i versi in questione dunque furono scritti proprio a Roma nel 64-65 e potrebbero quindi riflettere l'impressione delle spettacolari esecuzioni cui sembra riferirsi Giovenale. Certo, Giovenale non dice espressamente che quelle persone condannate a finire come torce umane fossero Cristiani, tuttavia la sua descrizione corrisponde fin nei dettagli agli spettacolari supplizî dei presunti colpevoli dell'incendio dell'Urbe nel 64; il riferimento a Tigellino poi fornisce una indicazione cronologica molto precisa e notevolmente circoscritta. Benché dunque il poeta non parli di Cristiani, se tuttavia l'episodio storico che aveva in mente era effettivamente quello del 64 , gli era certamente noto che i Cristiani ed essi soltanto erano stati incriminati come incendiarî e quindi messi a morte con le modalità descritte da Tacito, che in Giovenale trovano rimarchevole corrispondenza. Può essere interessante a questo proposito osservare che anche altrove Giovenale sembra presentare accenni alle persecuzioni perpetrate da un tiranno, questa volta Domiziano, contro diversi membri della classe dirigente di indirizzo stoicheggiante e contro i Cristiani: nella Satira IV il poeta deplora non solo la condanna a morte delle anime illustri di tanti senatori e notabili, ma anche e soprattutto -poiché a questo personaggio è riservata una attenzione del tutto particolare - la morte del senatore Acilio Glabrione (vv. 95-103), che fu condannato da Domiziano, oltre che per molitio rerum novarum (Suet. Dom. 10, 2), anche per ateismo e costumi giudaici (Dio LXVII 14), il che significa con ogni probabilità per Cristianesimo ${ }^{33}$. Anche qui, Giovenale non afferma esplicitamente che Acilio fosse cristiano, tuttavia era senz'altro al corrente della natura delle imputazioni a carico di Acilio stesso.

32 Lucano presenta crux anche a VI 545: Erittone «pendentia corpora carpsit abrasitque cruces»: evidentemente il supplizio della croce, cosi orribile, ben si confà all'episodio della maga tessala, tutto all'insegna dell'orrifico.

${ }_{33}$ Ivi Giovenale sembra riferirsi pure ai Cristiani chiamandoli cerdones (v. 153): si veda eventualmente il mio Giovenale, Sat. IV e il supplizio di s. Giovanni a Roma, che sarà pubblicato su «Gerión» (2000). Mi sia consentito esprimere tutta la mia gratitudine alla Prof. Marta Sordi che da anni segue con generosa e costante attenzione e con prodigalità di indicazioni le mie ricerche, alla Prof. Mirella Ferrari che con tanta cortesia mi ha dispensato i suoi preziosi consigli e alla Prof. Pilar Fernández Uriel per la sua davvero gentile disponibilità. 